\title{
The persistent influence of viewing environment illumination color on displayed image appearance
}

\author{
Trevor Canham, Marcelo Bertalmío; Universitat Pompeu Fabra; Barcelona, Spain
}

\begin{abstract}
Chromatic adaptation considering competing influences from emissive displays and ambient illumination is a little studied topic in the context of color management in proportion to its influence on displayed image appearance. An experiment was conducted to identify the degree to which observers adapt to the white point of natural images on an emissive display versus the color of ambient illumination in the room. The responses of observers had no significant difference from those of a previous experiment which was conducted with roughly the same procedure and conditions on a mobile display with a significantly smaller viewing angle. A model is proposed to predict the degree of adaptation values reported by observers. This model has a form such that it can be re-optimized to fit additional data sets for different viewing scenarios and can be used in conjunction with a number of chromatic adaptation transforms.
\end{abstract}

\section{Introduction}

In the content production industry, those responsible for mastering content to be distributed attempt to configure their viewing scenario to not only be reliable and consistent, but also to mimic the average conditions in which the content will ultimately be seen. For this reason, standards exist which dictate the parameters for display operation and mastering suite environmental setup $[1,2,3]$. While this paradigm may have been effective in the more homogeneous distribution landscape which existed at the time of its development, the only clear way it can be scaled to maintain its effectiveness in today's more varied market is via dynamic, device-side content adjustment methods. While a number of proprietary methods of this type have been developed and implemented by display manufacturers in their respective devices, these have been counterproductive to the creation of a standard color management ecosystem, as these parties view this as a way to differentiate their products rather than to preserve the creative intent of mastering artists. In addition, these systems only address specific parts of the problem and leave others out entirely. For example, image appearance shifts due to viewing conditions (ambient lighting [4], display field of view and size [5]) and observer perceptual variability [6], to name a few. A major reason for this is that the variability related to these factors has not been properly characterized (or even addressed in some cases) by the scientific community, which is explicable given that these involve complex interactions between human perception and imaging technology.

The topic of this writing, chromatic adaptation considering simultaneous influence from emissive displays and ambient illumination, can be included among the understudied. This problem focuses on identifying the degree to which observers adapt to the color of ambient illumination over the white point of the display. Here, we expand on an earlier study [7] which aimed to identify this for a mobile viewing scenario under a variety of viewing conditions. Our contributions include further experiments which add another set of tested conditions, this time using a display oc- cupying a significantly larger field of view. In the experiment a series of images are first displayed under a reference condition, where observers are instructed to take notice of the quality of their achromatic and memory color regions. Then, the observers are asked to adjust them back to this color balance from memory as they are displayed again under varying environmental conditions. To complete this task, observers use a 1D white balance control which shifts the color balance of images along a linear pathway connecting the values for the white point of the display to the color of the ambient illumination.

Using these data and those from the previous experiment, a model for predicting degree of adaptation in a motion picture viewing scenario is optimized and validated. This model takes a similar form to that which was included in CAT02 [8], and could potentially be used interchangeably with a range of chromatic adaptation transforms. The results of our additional tests show that even when the display is expanded far into the surround portion of the observer field of view, partial adaptation to illumination conditions persists to a similar degree compared to the mobile scenario.

\section{Related work}

As stated previously, a limited amount of research has been conducted on the topic of surround chromatic adaptation while viewing emissive displays. That which does exist presents conflicting results, revealing the extensive number of relevant factors which need to be accounted for in the problem. Several studies $[9,10,11]$ were conducted in the past decades addressing this topic for the application of soft copy/hard copy proofing. Each experiment involved observers performing an asymmetric matching or achromatic preference task involving natural or synthetic stimuli on an emissive display in a variety of viewing conditions. The results of these three studies averaged over all observers ranged from $60 \%$ to complete adaptation to the display while testing similar environmental conditions. There were several differences between the experiments which could potentially explain the discrepancy, including the observer task, the stimulus type, and the experimental cadence (adaptation times, etc.). This is evidence that degree of adaptation data cannot be reliably extrapolated to different viewing contexts unless these relevant factors are considered.

This work is an extension of [7], which aimed to characterize degree of adaptation to the display versus the surround for a mobile viewing scenario under six ambient lighting conditions (10000K, Illuminant A, and 2200K CCTs at brightness levels 5 and $102 \mathrm{~cd} / \mathrm{m}^{2}$ ). In this experiment, observers were presented a series of images ( 1 synthetic and 2 natural) on a mobile display under standard HDTV mastering environmental conditions $\left(\mathrm{D} 65,5 \mathrm{~cd} / \mathrm{m}^{2}\right)$ and were later asked to recreate the images via a 1D white balance control after adapting to the various illumination conditions. The control scheme was based on a von Kries [12] mixed-adaptation transform which shifted the white point of the image on screen along a vector in 1976 u'v' space con- 
necting the display and ambient white points. The results of the experiment demonstrated that the illumination color, brightness, and image content (synthetic vs. natural) were all statistically significant factors, leading to a range of results showing observer adaptation to be controlled between 26 and $90 \%$ by the display, considering experimental error tolerances.

The most widely recognized implementation of a deviceside, real-time ambient illumination correction scheme is the Apple True Tone system, which was recently addressed by Wu et al. in [13]. This writing describes a series of experiments which were performed to develop the system, as well as the practical details of its implementation. This included an experiment similar to [7] where observers were seated in front of a light booth and adjusted images on a display via a 1D white balance control to their achromatic preference. The stimuli set included natural images, application user interfaces, and text documents. A wide range of lighting conditions were tested including highly saturated colors, but a consistent luminance level was maintained. The results of each experiment condition were averaged and stored as a single degree of adaptation value in a look up table, allowing for a corrective method to be implemented for which a prediction for any given illumination color can be interpolated between tested points. Separate lookup tables were created for different viewing scenarios, demonstrating that they are specific to the conditions tested.

\section{Chromatic adaptation}

The oldest prevailing model for chromatic adaptation was proposed by Johannes von Kries [12]. It is calculated in the following way:

$$
I^{c}{ }_{a}=k^{c} I^{c}, k^{c}=\frac{1}{I^{c}{ }_{w}} \text { for } c \in L, M, S
$$

where $I^{c}{ }_{a}$ is the adapted cone response, $I^{c}$ is the original per channel cone excitation and $I^{c}{ }_{w}$ is the cone response to the scene white point. This states that the cone responses after adaptation are equal to the original responses scaled by those of the adapting stimulus. In reflection upon his theory, von Kries states that this is likely a simplification of a significantly more complex process. While further research proved this to be true, all chromatic adaptation models that have followed are expansions of the von Kries form which further address the complexity of the mechanism in various ways. In general, these extensions result in changes in the magnitude of predicted adaptation shifts while maintaining the direction of the von Kries prediction [14].

A significant finding confirming this greater degree of complexity comes from Hurvich and Jameson $[15,16]$ who identified the phenomenon of incomplete adaptation. In a study of factors affecting the perception of achromatic self-luminous fields, the two discovered that observers were more likely to identify a field with a D65 white point as achromatic at lower luminance levels than any other tested white point. The study also revealed that in the case of white points with a correlated color temperature of $2900 \mathrm{~K}$ and below, observers did not report the fields as appearing white at any luminance level. Along with evidence from our own visual experience (ie. we do not completely adapt to a pure red light source, for example, and perceive it as white over time), this illustrates the fact that our visual system is not able to adapt fully to any given illumination color, demonstrating the existence of some limiting processes in the visual pathway.

Later works from Fairchild [17] and Zhai [18] further explore this concept by comparing its effects in the context of emissive and illuminated stimuli. The results showed that observers were capable of adapting more completely to a range of colored lighting when viewing illuminated stimuli, implying that our visual system is more capable of "discounting the illuminant" and maintaining color constancy in this scenario than it is when viewing emissive or self-luminous stimuli (like electronic image displays). This shows that even our adaptation processes which occur sub-consciously make a distinction between real environments and ones reproduced by imaging systems.

The multi-faceted and universal nature of adaptation processes is well characterized in the recent review from Webster [19]. Within, the author pulls examples from the large body of work on visual adaptation to show that adaptive processes happen at all stages of the visual system and thus are a combination of long term, high level cognitive and near-instantaneous low level physiological processes. To illustrate, Webster calls upon the example of luminance adaptation, citing how it combines instantaneous gain shifts in the retina along with cognitive adaptation considering a pool of reference points from multiple surfaces and fixations. In doing this, the visual system characterizes the average illumination in the environment allowing for color constancy to be enforced in response to lighting changes. This implies, however, that an element of memory and an extended adaptation time course must be involved in the process. It is hypothesized that chromatic adaptation behaves in a similar fashion [17].

In order to describe these processes with a simplified set of input parameters, various groups have proposed the use of degree of adaptation functions to be included alongside practical chromatic adaptation transforms. These functions return an "effective" white reference point $I_{w}$ for which incomplete adaptation has been considered. This new reference white can then be passed to any simple chromatic adaptation transform such as that of von Kries, making it a modular and simple solution for predicting these complex phenomena. While the first of this type of consideration was proposed by Hunt [20], the most well-known is that which is included in the chromatic adaptation step of CIECAM02 [8]. This function is limited, however, as it only considers the luminance of the adapting stimulus and not its color, which Hurvich and Jameson clearly show is relevant. Thus, extensions have been proposed which also include the parameters of illumination color and saturation along with luminance, such as that of Lee et al. [21].

\section{Methods}

The goal of this experiment is to determine the impact of surround chromatic adaptation on the color appearance of image content viewed on emissive displays. As demonstrated by the studies cited above, this requires at the very minimum some number of observers to perform an achromatic preference or matching task. In the spirit of testing scenarios that are as close as possible to the application of aesthetic image evaluation, the chosen stimuli are natural images viewed on a common reference monitor. The images are first displayed under the SMPTE standard HDTV mastering environmental conditions as a control (D65, 5 $\mathrm{cd} / \mathrm{m}^{2}$ ), where observers are instructed to take note of the general image color balance and the appearance of specific memory color/achromatic regions. Then the observers are asked to adjust them back to this color balance from memory as they are displayed again under varying environmental conditions.

This adjustment is executed via a $1 \mathrm{D}$ control scheme which alters a mixed adaptation weighting factor $d$ in a simple von Kries based transform (Eq. 1). The concept of mixed adaptation is used to describe scenarios in which multiple white references exist in a scene. This involves finding a new effective $I_{w}$, which is determined as some weighted ratio between the chromaticity values 
of the two references, calculated as follows:

$$
I_{w}=d I_{\text {display }}^{c}+(1-d) I_{\text {ambient }}^{c}
$$

Thus, the control scheme is a discretized adjustment of image color balance on some straight line vector in 1976 u'v' chromaticity space which intersects the color of a given ambient condition and the display white point. Thus, it should be noted that observer responses will be reported as the chosen mixed adaptation ratio $d$, for which a value of 1 indicates complete adaptation to the D65 display white point.

To verify that the $1 \mathrm{D}$ control was appropriate to describe the adapted states of observers, an abbreviated version of the experiment was administered to professionals (colorists from the cinema industry.) In this version of the experiment, the observers were permitted a second dimension of control which would shift the white point perpendicularly to the $1 \mathrm{D}$ control vector. Through post-experiment discussions, the participants reported that they could determine an acceptable result given their memory of the reference without the use of the second dimension of control. This corroborates the hypothesis that the von Kries mixed adaptation transform is acceptably accurate in predicting the direction of observer chromatic adaptation, allowing observers to report their adapted state using the control scheme within a visually acceptable range of error.

This experiment not only includes ambient illumination chromaticity as a variable, but also image content, display/ambient brightness ratio, and image starting color balance are varied between trials (Table 1). Environmental illumination was varied between three chromaticity and two brightness levels. Three natural images were used, which were graded under the reference environmental conditions of the experiment to have equal primary values for their diffuse white portions and also to have an appearance similar to what might be seen in television or cinema content (figure 1). Images are also presented to observers three times for the same conditions with starting color balances at different points along the control vector to obtain an average between presentation biases. The combination of these variables results in a total of 54 observations per observer during the body of the experiment.

Table 1: Experimental factors.

\begin{tabular}{c|c|c}
\hline Factor & Type & Levels \\
\hline Ambient color & Varying & III A, 2200K, $1800 \mathrm{~K}$ \\
\hline Ambient luminance & Varying & $5 \mathrm{~cd} / \mathrm{m}^{2}, 102 \mathrm{~cd} / \mathrm{m}^{2}$ \\
\hline Image content & Varying & 3 natural images \\
\hline Starting image balance & Varying & Cool, Middle, Warm \\
\hline Display luminance & Constant & $100 \mathrm{~cd} / \mathrm{m}^{2}$ \\
\hline Viewing angle & Constant & $50 \times 28$ Degrees $(27$ ' diag) \\
\hline User task & Constant & Memory Matching \\
\hline
\end{tabular}

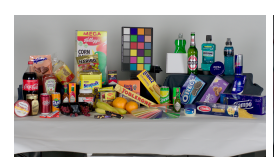

(a)

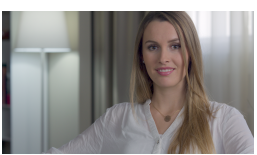

(b)

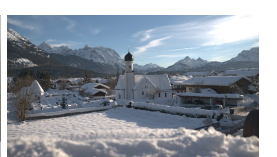

(c)
Figure 1: Experimental Images. Provided by ARRI camera systems for the HDR4EU project.

The experiment is carried out in the IP4EC mastering lab at Universitat Pompeu Fabra in Barcelona, Spain. The lab is equipped with two Arri Sky Panel s30-c LED fixtures which illuminate the back wall of the lab. The LEDs work on a four primary system which is capable of producing light of high spectral quality and dynamic range [22]. The lights are controlled with 8-bit drive values transmitted via art-net DMX signals using the
Open Lighting Architecture (OLA) framework. OLA commands are called by a Matlab Psychophysics toolbox [23] test bed which also displays images according to experimental cadence and observer input. Chromatic adaptation transforms were applied to images following observer input using pre-calculated 3D LUTs. Images generated from the workstation (Late 2018 Mac book pro running OSX 10.8.5) are displayed via HDMI connection on a Sony PVM-A250 reference monitor calibrated to Rec. 709 primaries and a D65 white point. Observers are seated at a viewing distance of two picture heights from the surface of the display resulting in a 50x28 degree viewing angle for the 16:9 full $\mathrm{HD}$ screen, reflecting a real-world mastering scenario. The ambient illumination of the room was characterized using a Photo Research PR 705 Spectrophotometer. Measurements were taken with the device aimed at the wall just above the display. Extra measurements were taken around the screen to confirm a degree of illumination uniformity on the back wall.

Prior to the experiment all observers were screened for color discrimination deficiencies and briefed with experimental instructions aloud. Next, they were led into the experiment area and were given two minutes to adapt to the reference environmental conditions. During each adaptation period, observers were encouraged to shift their gaze around the room, taking notice of familiar objects like their skin, clothing and belongings. The test images were then presented in their native color balances and observers were given as much time as they liked to memorize the appearance of the achromatic and memory color scene elements of each one. Next, observers were asked to complete a brief training period introducing them to the experiment task. The memorization period was then repeated for reinforcement. Following this, the body of the experiment was carried out. The order of environmental conditions was randomized between observers to avoid any bias in the results caused by the proximity of the trial to the memorization period. After adapting to each environmental condition, observers were presented with the three images from the memorization phase in three different initial color balances each. Observers were asked to adjust the presented image back to their memorized color balance using the control scheme described above.

A total of 15 observers $(11 \mathrm{M}, 4 \mathrm{~F})$ participated in the experiment. Their ages ranged from 23-39 with a mean age of 28.6. Observers were compensated for their participation.

\section{Results}

Average observer mixed adaptation ratios between display white point and ambient illumination chromaticities are plotted with 95\% confidence error bars in Figures 2 and 3. An ANOVA test showed no significant difference when comparing the mean degree of adaptation results between the different ambient illumination colors $(\mathrm{p}=0.1285)$. As in the previous experiment, a significant difference was shown between the user response distributions to the two brightness levels $(\mathrm{p}<0.0001)$ as well as between the images tested $(p=0.0174)$.

\section{Corrective Model}

In a similar fashion to $[8,21]$ we propose a degree of adaptation model considering the illumination color and luminance. This model is intended to be used for the specific case of adjusting electronically displayed natural images to account for ambient viewing conditions, and takes the following form:

$$
d=1-\left(\alpha \exp (-x)+\beta\left(L_{a} / L_{d}\right)\right)
$$

Here, a value of $d=1$ would indicate that the observer is fully adapted to the white point of the display. It takes as input the 


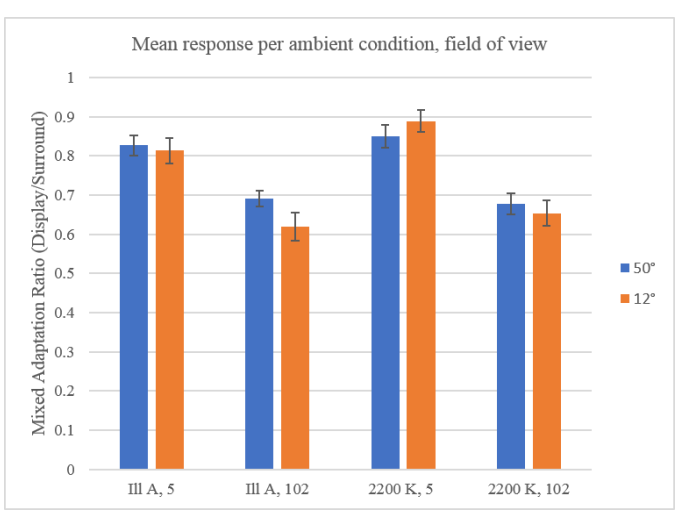

Figure 2: Experimental results per condition tested, compared to results of [7] for common conditions tested, reported as degree of adaptation to the display.

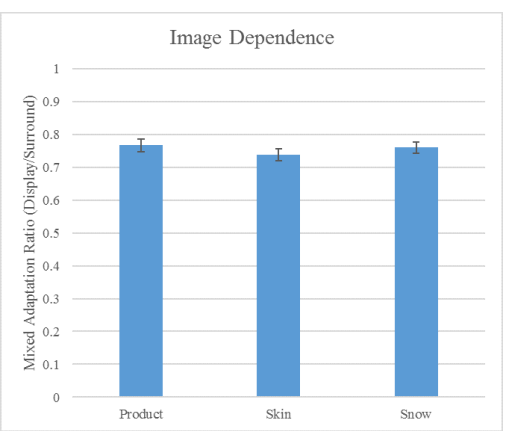

Figure 3: Experimental results per image tested, reported as degree of adaptation to the display.

ambient/display luminance ratio $\left(L_{a} / L_{d}\right)$ and illumination color (specifically its delta u'v' [Du'v'] distance $x$ from the monitor white point.) Each factor is fitted with a scalar coefficient which can be optimized to best fit the data for the conditions tested. The function returns a degree of adaptation value $d$ which can be used with Eq. 2 to determine the 'effective' adapting stimulus $I^{c}{ }_{w}$ as far as mixed/incomplete adaptation is concerned, encoding the effects of high-level cognitive processes. This new adapting stimulus value can then be passed to a chromatic adaptation transform such as that of Eq. 1.

An optimization process was performed to find the proper coefficient values for $\alpha$ and $\beta$. The optimization function aimed to minimize the Du'v' value between the color corresponding to the degree of adaptation value predicted by the model and that which was reported by the observers. We found optimal coefficient values $\alpha=0.1529$ and $\beta=0.2419$, training for the data set from [7]. Using these coefficients the function predicts the responses of the observers from the experiment described here. The Du'v' values for both sets are reported in Table 2.

Table 2: Minimum, mean, and maximum Du'v' prediction errors for optimization and test sets using the reported coefficients.

\begin{tabular}{c|c|c}
\hline Error & Optimization & Test \\
\hline Min & $<0.001$ & $<0.001$ \\
\hline Avg & 0.007 & 0.009 \\
\hline Max & 0.023 & 0.024 \\
\hline
\end{tabular}

While it may seem more appropriate to use a perceptual difference metric like DeltaE for an error function, the use of this metric requires the specification of an adapting stimulus, and in this case we are looking at the difference between adapting stimulus values. However for perspective, if we take the delta E 2000 value for the worst prediction from the test set, taking D65 as the white point, we find an error of 2.2 .

\section{Discussion}

Despite the shift in display field of view between this experiment and the one previously reported in [7], if we compare the similar lighting conditions for the previous experiment (isolating for natural images) a one-way ANOVA comparison returns no significant difference $(p=0.6375)$. Figure 2 compares mean responses for each condition between the two field of view settings. For three of the four viewing conditions, the $95 \%$ confidence error bars overlap, and neither viewing angle results in a consistently higher or lower degree of adaptation result than the other. A possible explanation for this could be that that our adaptive processes always maintain some consideration for the current illumination (if any exists) of the environment which we identify ourselves being located within, even if we are focusing on an emissive display.

Aside from the ways in which this particular experiment deviates from being a perfect simulation of an observer motion picture viewing scenario, we know that the results likely cannot be generalized to other viewing scenarios, like user interfaces or ebooks for example. For this reason, the proposed model incorporates the scalar coefficients $\alpha$ and $\beta$ which can be retrained for different observer data sets. This can be done in the same way as was described above, by searching for new coefficient values which minimize Du'v' error between observer responses and model predictions.

\section{Conclusion}

An experiment was conducted identifying the degree to which observers adapt to the white point of natural images on an emissive display vs. the color of ambient illumination in the room. The results were general for all ambient illumination colors tested, and were similar to those of the previous experiment which was conducted with a drastically reduced viewing angle for roughly the same conditions. A possible explanation for this could be that that our adaptive processes maintain some consideration for the current environmental illumination (if any exists) whenever we view emissive displays, with little regard to the amount that the display occupies the observer field of view. Using these data and those from the previous experiment, a degree of adaptation model was formulated, and optimized. This model has a form such that it could be re-optimized to fit any different or additional data, for instance an expanded set with a larger number of tested environmental conditions.

\section{Acknowledgments}

The authors would like to thank all observers for their participation, in particular the colorists from Deluxe Spain who generously lent their time and spectrophotometer to the experiment. This work has received funding from the European Union's Horizon 2020 research and innovation programme under grant agreement number 761544 (project HDR4EU) and under grant agreement number 780470 (project SAUCE), and by the Spanish government and FEDER Fund, grant ref. PGC2018-099651-B-I00 (MCIU/AEI/FEDER, UE).

\section{References}

[1] SMPTE ST 2080-1:2014, "Reference White Luminance Level and Chromaticity for HDTV."

[2] SMPTE RP 2080-2:2014, "Measurement and Calibration Procedures for HDTV Display Luminance Levels and Chromaticity."

[3] SMPTE ST 2080-3:2017, "Reference Viewing Environment for Evaluation of HDTV Images." 
[4] C. James Bartleson and Edwin J. Breneman. 1967. "Brightness perception in complex fields", JOSA, 57(7):953-956.

[5] Alexandre Chapiro, Timo Kunkel, Robin Atkins, and Scott Daly. 2018. "Influence of Screen Size and Field of View on Perceived Brightness", ACM Transactions on Applied Perception.

[6] Abhijit Sarkar, Laurent Blonde, Patrick Le Callet, Florent Autrusseau, Patrick Morvan, Jurgen Stauder. 2010. ”Toward Reducing Observer Metamerism in Industrial Applications: Colorimetric Observer Categories and Observer Classification", 18th Color Imaging Conference Final Program and Proceedings.

[7] Trevor Canham, Micheal J. Murdoch, David Long. 2018. "Influence of Ambient Chromaticity on Portable Display Color Appearance", SMPTE 2018 Annual Technical Conference Exhibition.

[8] CIE. 2004a. "A Colour Appearance Model for Color Management Systems: CIECAM02", CIE TC8-01 Technical Report, CIE Pub. No. 159.

[9] Mark E. Gorzynski. 1992. "Achromatic perception in color image displays", Thesis, Munsell Color Science Laboratory, Rochester Institute of Technology.

[10] Sharron Henly. 2000. "Quantifying mixed adaptation in crossmedia color reproduction",Thesis, Munsell Color Science Laboratory, Rochester Institute of Technology.

[11] Naoya Katoh. 1998. "Effect of ambient light on the color appearance of softcopy images: Mixed chromatic adaptation for selfluminous displays”, Journal of Electronic Imaging 7(4), 794-806, October 1998.

[12] J. von Kries. 1902. "Chromatic Adaptation", Festschrift der Albrecht-Ludwig-Unversitat, Fribourg, [English translation: D. L. Mac-Adam, Sources of Color Science, MIT Press, Cambridge, 1970].

[13] Jiaying Wu, Lu Zhang, Serhan Isikman, Cheng Chen. 2019. "Enhanced Viewing Experience Considering Chromatic Adaptation", SID 2019 Digest, 61-1, 857-860.

[14] Tushar Chauhan et al. 2014. "The achromatic Locus: Effect of navigation direction in color space", Journal of Vision; 14(1):25, 1-11.

[15] Leo M. Hurvich and Dorothea Jameson. 1951a. "A Psychophysical Study of White. I. Neutral Adaptation", Opt. Soc. Am., 41, 521-527.

[16] Leo M. Hurvich and Dorothea Jameson. 1951b. ”A Psychophysical Study of White. HI. Adaptation as Variant", Opt. Soc. Am., 41, 787801 .

[17] Mark Fairchild. 1992. "Chromatic Adaptation to Image Displays", TAGA 2, 803-824.

[18] Qiyan Zhai, M. Ronnier Luo, Peter Hanselaer, Kevin A.G. Smet. 2016. "Modelling Incomplete Chromatic Adaptation and Colour Contrast Using Memory Colour", Color and Imaging Conference.

[19] Michael A. Webster. 2015. "Visual Adaptation", Annu. Rev. Vis. Sci. 1:547-67.

[20] Robert W.G. Hunt. 1987b. "A Model of Colour Vision for Predicting Colour Appearance In Various Viewing Conditions", Col. Res. Appl., 12, 297-314.

[21] Sung-Huk Lee, Myong-Hwa Lee, Kyu-Ik Sohng. 2008. "Factors of Incomplete Adaptation for Color Reproduction Considering Subjective White Point Shift for Varying Illuminant", IEEE Trans. Fundamentals, vol. E91-A, No. 6, June 2008.

[22] Matthew Allard ACS. 2016. "Hands on with the Arri Skypanels - a single operater's perspective", News Shooter, Online: https://www.newsshooter.com/2016/05/18/hands-on-with-thearri-skypanels-a-single-operators-perspective/

[23] David H. Brainard. 1997. "The Psychophysics Toolbox", Spatial Vision; 10:433-436.

\section{Author Biography}

Trevor Canham was born and raised in Rochester, NY, where he went on to receive his B.S. in Motion Picture Science from the Rochester Institute of Technology in 2018. Since that time he has been working as a research engineer on dynamic color management systems within the Image Processing for Enhanced Cinematography group at Universitat Pompeu Fabra in Barcelona, Spain. His interests lie in the interaction between human perception and aesthetic imaging systems.

Marcelo Bertalmío (Montevideo, 1972) is a full professor at Universitat Pompeu Fabra, Spain, in the Information and Communication Technologies Department. He received his Ph.D. in electrical and computer engineering from the University of Minnesota, USA, in 2001. His current research interests are in developing image processing algorithms for cinema that mimic neural and perceptual processes in the visual system, and to investigate new vision models based on the efficient representation principle. 